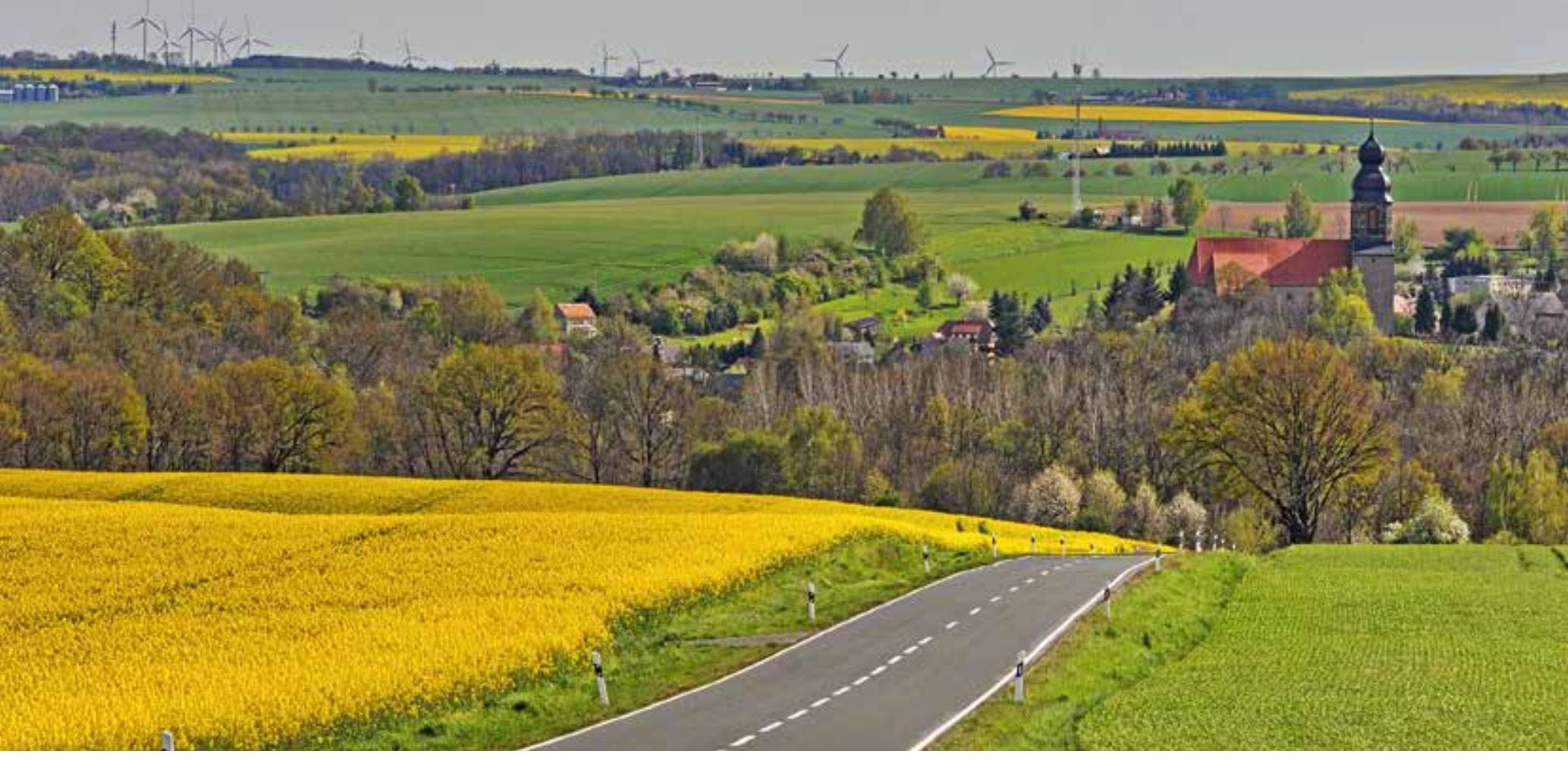

\title{
Sehenswürdigkeiten der Natur in der Lommatzscher Pflege
}

\section{Frank Ende}

Die Lommatzscher Pflege wird in der romantischen Literatur als die „Toskana des Nordens“ bezeichnet. Sie ist durch sanfte Hügel und liebliche Bachtäler wie das Jahnatal oder das Käbschütztal, Streuobstwiesen, Baumbestände an Wege und Straßen sowie kuschlig in Senken eingebettete Dörfer gekennzeichnet. Andere sehen in ihr eine ausgeräumte Agrarsteppe, in der sich endlose Felder ohne Gehölzstrukturen und Wiesen ausdehnen und in der die Tiere und Pflanzen keinen Lebensraum haben und schon manche Arten verschwunden sind. Gert Lippold vom Schloss Scharfenberg nennt es die Schaffung einer „tadellos optimierten Agrarwüste“.

Was ein Wald ist, da kann man geteilter Meinung sein, und wenn man mit Menschen aus waldreichen Gegenden Sachsens unterwegs ist, gibt es dazu auch unterschiedliche Sichtweisen. Jedenfalls ist das Schleinitzer Großholz das größte zusammenhängende Waldgebiet der Lommatz-
scherPflege, welchesinderOst-West-Ausdehnung gerade einmal 1500 Meter und in der Nord-SüdAchse 700 Meter misst. Nur ein halbe Stunde braucht man, um es zu durchlaufen.

\section{Naturschutzgebiet Schleinitzer Großholz und gleichnamiges FFH-Gebiet}

Größe: Naturschutzgebiet 14,65 Hektar, Gesamtgebiet 52,4 Hektar

Lage: Das Gebiet liegt etwa 5 Kilometer südwestlich von Lommatzsch und ca. 2 Kilometer von Schleinitz entfernt, genau an der Kreisgrenze zum Landkreis Mittelsachsen. Ein markanter Höhenpunkt ist die Schleinitzhöhe.

Schutzzweck: Erhaltung und Wiederherstellung der natürlichen Bestockung im größten und landschaftsprägenden Laubwaldkomplex der waldarmen Lommatzscher Pflege als naturtypisches Refugium für Pflanzen- und Tierarten und landschaftskundliches Studienobjekt.
Blick von den Pappelweiden in das Ketzerbachtal und auf den Ort Leuben

Foto: Gerhard Schlechte 


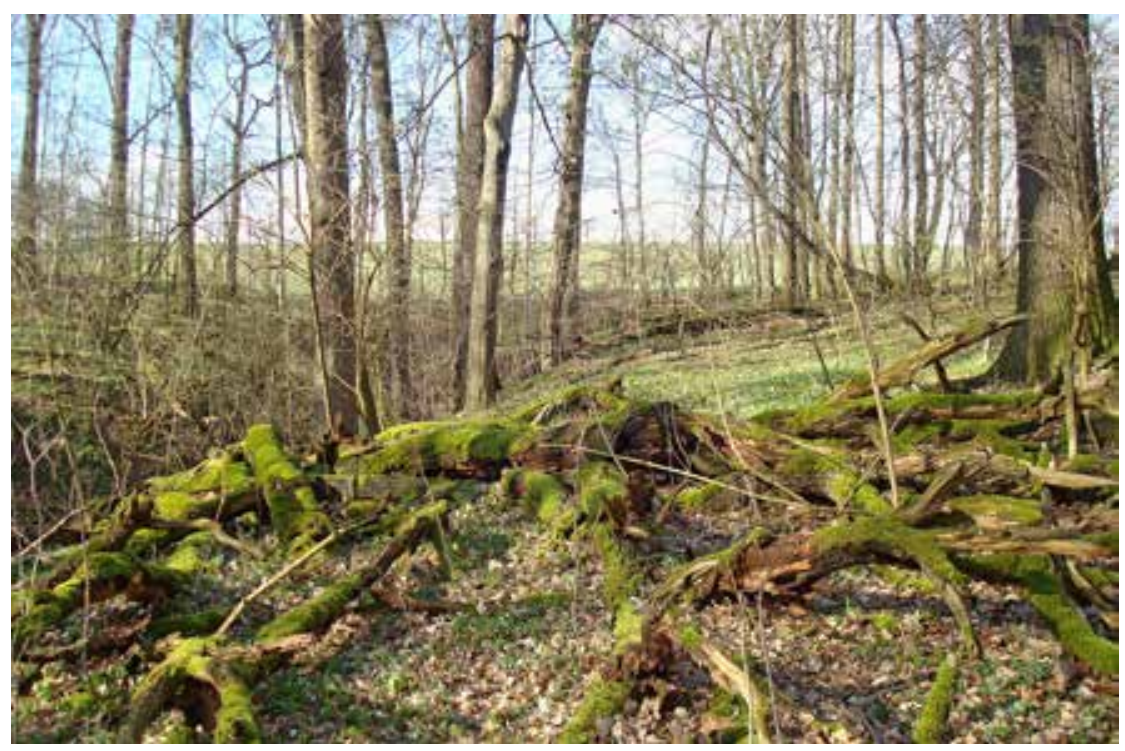

Frühling im Großholz Foto: Frank Ende

Märzenbecher im Feldgehölz von Petzschwitz Foto: Frank Ende
Das Großholz ist eine artenreiche Waldinsel inmitten einer großflächigen Agrarlandschaft und somit Rückzugsraum für zahlreiche Tier-und Pflanzenarten. Der Wald gehörte ehemals zum Rittergut Schleinitz. Er war 1761 etwa 130 Hektar groß und besaß zahlreiche Verbindungen zu angrenzenden Feldgehölzen und Wiesentälern der umliegenden Dörfer. Um 1850 war das Großholz zu einer Waldinsel geworden. Anfang des 20. Jahrhunderts nahm die Familie von Friesen aus wirtschaftlichen Gründen umfangreiche Rodungen vor, was die Größe enorm verringerte. Das Naturschutzgebiet kann in einen Rundweg über Feldwege von Schleinitz aus erwandert werden.

Der Wald stellt sich als Eichen-Hainbuchen-Gesellschaft und Waldmeister-Buchenwälder dar. Es wachsen Stiel- und Traubeneiche, Winterlinde, Rotbuchen, Berg-Ahorn und auf einigen Standorten auch Esche und Birke.
Sehenswert ist der Frühlingsaspekt des Großholzes, beginnend mit der Blüte des Märzenbechers, des Hohlen Lerchensporns, des Lungenkrauts und des Buschwindröschens. Besonders hervorhebenswert sind die ausgedehnten Bestände des Märzenbechers, die allerdings schwer zu finden sind. Für den Naturliebhaber besser erschlossen ist eines der bedeutendsten Märzenbechervorkommen Sachsens im nahe gelegenen Petzschwitz. Hier ist großflächig in einem Feldgehölz fast vollständig der Boden mit den Blüten der Märzenbecher bedeckt. Dieses Vorkommen kann es durchaus mit den Polenzwiesen in der Sächsischen Schweiz aufnehmen. Weitere Märzenbecherflächen kann man gut in einen kleinen Park in Kiebitz bei Ostrau und im Park von Jahnishausen an der Jahna bewundern.

Die Flora ist mit 136 Arten, davon 37 Baumund Straucharten, 118 Krautige und 8 Moosen, vertreten. Bei den Säugetieren sind besonders die Sommerquartiere der beiden Fledermausarten Großes Mausohr und Mopsfledermaus hervorzuheben, wobei die letztere vom Aussterben bedroht ist. Bei den 86 Vogelarten ist auf die beiden Milanarten Rot- und Schwarzmilan, auf die Brutvogelvorkommen von der streng geschützten Arten Schafstelze, Sperbergrasmücke, Braunkehlchen und Schwatzkehlchen und die fünf Spechtarten zu verweisen.

Trotz der Ausweisung als Naturschutzgebiet seit 1961 blieben auch die starken Waldnutzungen mit Holzeinschlägen und Feuerholzgewinnung bis in die heutige Zeit erhalten. Probleme bereitet auch die starke Bodenerosion durch Wasser, da das Naturschutzgebiet an vielen Seiten an Ackerschläge angrenzt. Auch werden die Waldsäume bis in den Traufbereich der Bäume für eine maschinengerechte Bewirtschaftung der Ackerflächen regelmäßig beschnitten, so

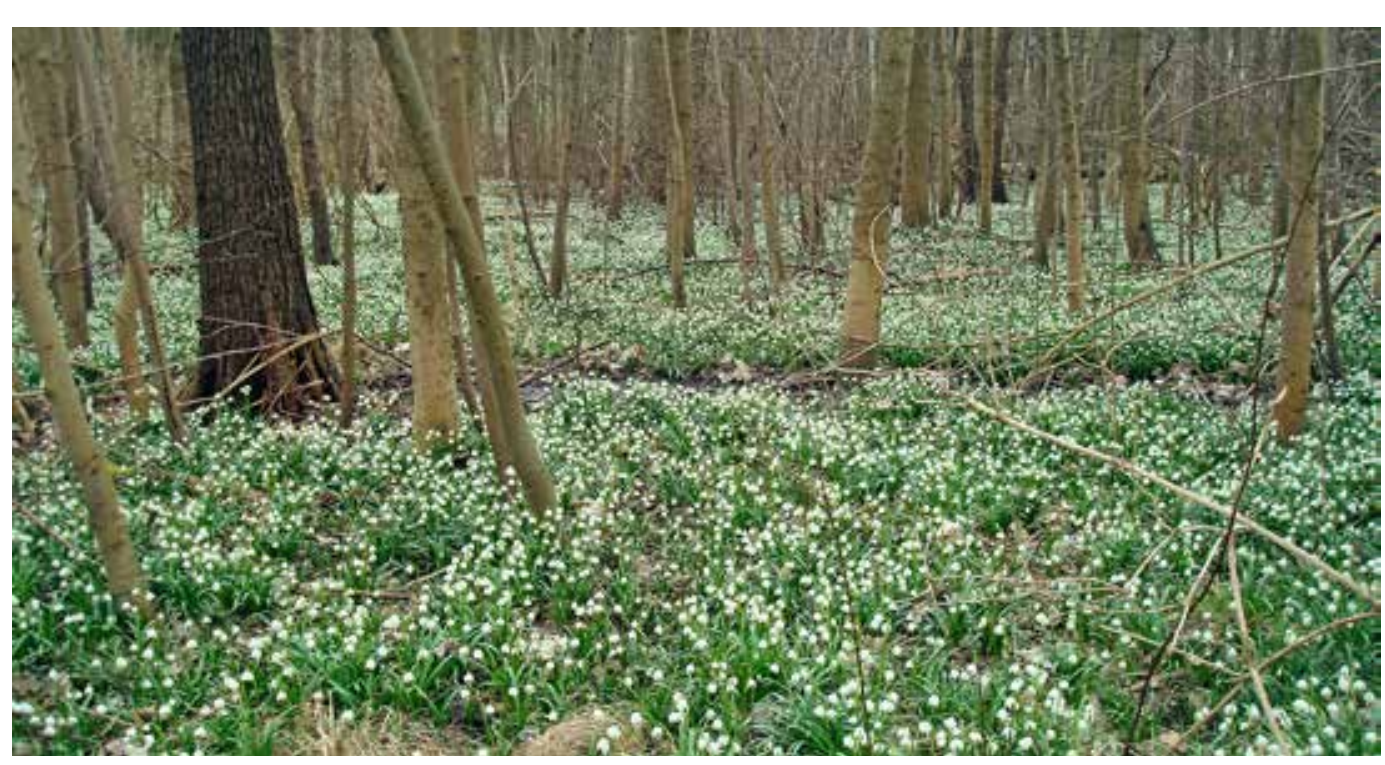


dass eine Pufferzone zum Feld fehlt.

Nur wenige Autominuten vom Großholz entfernt liegt im nördlichen Teil des Landkreises Mittelsachen an der Grenze zu den Landkreisen Meißen und Nordsachsen in Ostrau ein Naturschutzgebiet ganz anderer Art. Es entstand aus der intensiven Kalkbergbautätigkeit der vergangenen Jahrhunderte. Schon in der jüngeren Vergangenheit hatte man hier mehrere Flächennaturdenkmale angelegt.

\section{Naturschutzgebiet Alte Halde - Dolomitgebiet Ostrau}

\section{Größe: 26,5 Hektar}

Lage: Das Naturschutzgebiet liegt ein bis zwei Kilometer südöstlich des Ortes Ostrau und besteht aus zwei Teilen, dem Gebiet Bruch Münchhof und dem Gebiet Alte Halde, Dolomitwand und Trockenhang Zschochau

Schutzzweck: Sicherung eines Landschafsausschnittes mit anstehendem Zechsteindolomit als überregionale Besonderheit. Erhaltung und Entwicklung der Flora und Fauna insbesondere kalk- und basenreicher sowie wärmegetönter Standorte und eines naturnahen Bachabschnittes mit Bachauenwald. Rückzugsgebiet vieler Arten in strukturarmer Agrarlandschaft.

An mehreren Talstellen trat der Kalk offen zutage. Diese Gesteinsvorkommen des plattig abspaltenden Kalksteins wurden schon im frühen Mittelalter von den Daleminziern für die Wallbefestigungen ihrer Burganlagen in Hof/Stauchitz und Zschaitz genutzt. Dies zeigen die Funde auf den beiden Burganlagen, die man noch heute auf den Feldern finden kann. Es ist zwar nicht in den Akten belegt, aber sicher haben die Mönche aus Altzella den Kalk in der Flur ihres Vorwerks Münchhof abbauen lassen und nach

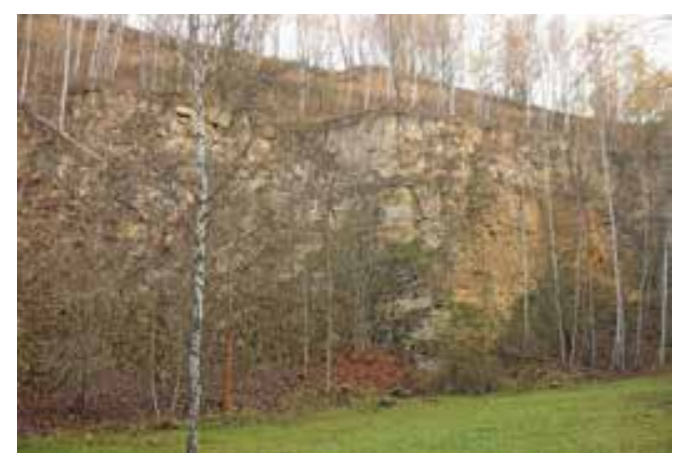

Blick von der Staatsstraße auf das Gebiet Alte Halde und das angrenzende heutige Kalkwerk Foto: Frank Ende
Nossen transportiert. Dies belegen noch heute einige Flurnamen für Wege als Kalkstraße. Der Kalkabbau zog sich von Ostrau-Münchhof über Kiebitz, Rittmitz, Schrebitz bis in die Gegend von Mügeln.

Wenn man bedenkt, dass in dem heutigen Naturschutzgebiet 1945 noch betriebsbereite Kalkbrüche vorhanden wurden, in den der Abbau nach wenigen Jahren zugunsten eines konzentrierten Kalkabbaus auf dem Gebiet des heutigen Kalkwerkes Ostrau aufgegeben wurde, ist die großartige Naturentwicklung nicht genug zu würdigen. In den ehemals klein strukturierten Kalkbrüchen waren genüg Biotope vorhanden, deren Pflanzen und Tiere das Gebiet des aufgegebenen Kalkabbaus besiedelten. Von 1986 bis 2004 wurden Rechtsstreite mit dem Besitzer der Ostrauer Kalkwerke geführt. Dieser wollte eine fast freistehende Dolomitwand im Schutzgebiet mit einer Million Tonnen Kalkstein bei einer Abraummächtigkeit von nur acht bis zehn Metern abbauen. Er verlor die Prozesse und die Wand steht heute noch. Das erst 1999 eingerichtete Naturschutzgebiet hat weiter Bestand.

Ein bedeutendes Winterquartier der sehr seltenen Mopsfledermaus befindet sich in zwei ehe-

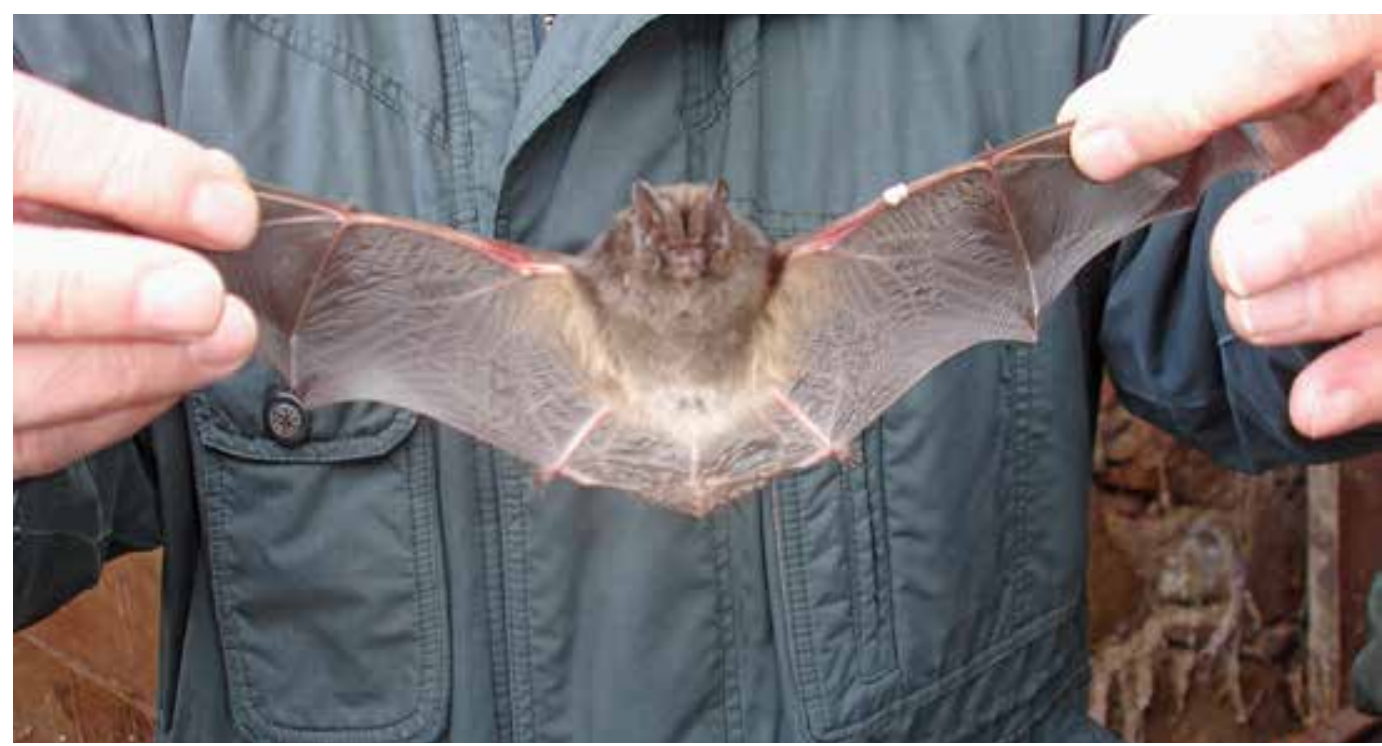

Mopsfledermaus bei der Winterkartierung Foto: Frank Ende 
Historische Ansicht des Kalkwerkes im Tännigt - Sammlung Frank Ende maligen Verbindungsstollen vom Kalkbruch zu den Kalköfen in Münchhof. Hier werden in jedem Winter von Fachleuten die Anzahl der Tiere erfasst. Neben der Mopsfledermaus wurde auch das Große Mausohr, das Braune Langohr und die Fransenfledermaus gefunden. Die Tiere überwintern in den Spalten zwischen den Bruchsteinen an der Tunneldecke.

Bei der Fauna muss unbedingt auf die Schneckenvorkommen verwiesen werden. Schon in den 1970er Jahren wurden hier die Schnecken erfasst und als für Sachsen bedeutendes Vorkommen eingeschätzt. 39 Molluskenarten sind hier bekannt und von den Schnecken mit ihren 37 Arten sind drei in Sachsen stark gefährdet. Die Große Laubschnecke hat hier den östlichsten Punkt ihrer Verbreitung in Sachsen und die Weiße Heideschnecke kommt nur noch an einer weiteren Stelle in Sachsen vor.

Bei der Flora wurden 266 Arten nachgewiesen. Es sind vor allen Arten, die kalkreiche Standorte lieben. Dies ist für ein solch kleines Naturschutzgebiet eine beachtliche Artenzahl, die sich durchaus mit anderen Naturschutzgebieten in Sachsen messen kann.

Beide Teile des Naturschutzgebietes verdanken ihre Entstehung dem Kalkbergbau, der die Landschaft formte. Im Gebiet Alte Halde ist eine Dolomitwand $\mathrm{zu}$ besichtigen. Am Fuße dieser Wand gedeihen vor allem die Nähstoffanzeiger wie Brennnessel und Schwarzer Holunder. Zukünftig wird sich hier die Flora schluchtwaldartig entwickeln. Die unmittelbar angrenzende Abraumhalde ist mit einem Eichen-Hainbuchenwald bestockt. Hier sind auch Buschwindröschen, Aaronstab, Maiglöckchen, Türkenbund-Lilie, Wald-Ziest und Waldveilchen zu finden. Der Trockenhang ist eine südexponierte Fläche mit zum Teil mageren Hangwiesen und einer Streuobstwiese. Hier sind Hain-Salbei, Zypressenwolfsmilch, Rotes Straußengras und mehrere Orchideenarten zu finden. Der Birmnitzbach in der Aue hat eine weitestgehend natürliche Morphologie mit einem stark gewundenen Lauf. Die Wiesen der Bachaue sind stark eutrophiert. Der Bereich Münchhof ist sich in seiner Entwicklung weitestgehend selbst überlassen gewesen. Die Reste eines Pistolenschießstands aus den 1970er Jahren sind noch erhalten. So stellt sich der Kernbereich des Bruches als wertvolles naturnahes Mosaik von Sukzessionsstadien dar. Hier befindet sich auch eine südexponierte offene Felswand. Auf der trockenen Magerwiese davor wachsen verschiedene Orchideenarten, Wiesenschlüsselblume und Kartäusernelke. Im Steinbruchkessel befindet sich ein Birkenwald. Ein schluchtwaldartiges Teilstück weist ein reichhaltiges Vorkommen des Braunen Storchschnabels und des Leberblümchens auf.

Das Naturschutzgebiet kann man, aus dem Jahnatalwanderweg kommend, erkunden. Der Bruch Münchhof ist zwar nicht begehbar, aber die davor vorhandenen Kalköfen sind eine Besichtigung wert, ebenso die benachbarten Kalköfen im Kalkgrund Ostrau. Von hier aus geht es mit dem Rad am besten über Zschochau oder zu Fuß am Kalkofen Ostrau Dresdner Straße vorbei zur Alten Halde.

Von Zschochau auf einen Feldweg in Richtung Pulsitz an einem weiteren Schutzgebiet vorbeikommend, erreicht man das Flächennaturdenkmal Tännigt. Das Schutzgebiet hat nur eine Größe von 3,2 Hektar und ist ein ehemaliger Kalkbruch. Der früheste Nachweis des Kalkabbaus für den Tännigt ist ein Gesuch von Johann Georg Eulitz aus Pulsitz um Konzession zur Anlegung eines Kalkofens aus dem Jahr 1758.

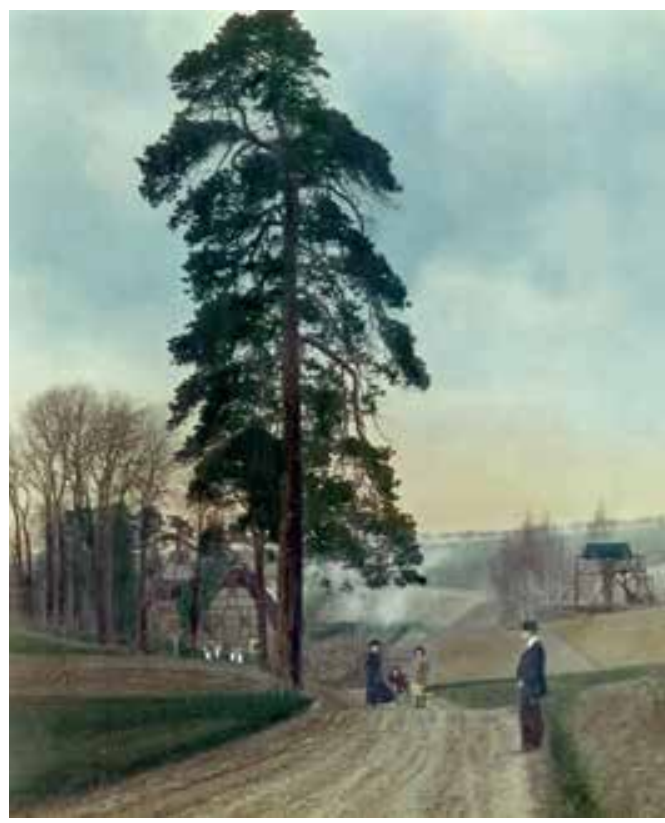

Floristisch ist hier das Vorkommen einer Orchideenart, des Großen Zweiblatts, von großer Bedeutung. Neben dem Bruch zieht sich eine Erosionsrinne in das Tal der Jahna hin. Diese Rinne war früher der Fahrweg zum Transport des Kalkes in das Jahnatal und wurde von Bauern landwirtschaftlich als Wiese genutzt. Die Fläche liegt völlig isoliert inmitten eines Ackerkomplexes von etwa 370 Hektar in der Landschaft. Im Meilenblatt ist hier 1821 noch eine reich strukturierte Landschaft mit Rainen, Wiesen, Gehölzen und Wegen mit Flurnamen wie Kaisertisch, Toter Mann und Steudtner Loch dargestellt. Dieses ist heute alles verschwunden. Der letzte Weg und eine Wiese wurden nach 1990 zum Teil mit Raupen beseitigt. 
Erst 2011 wurde nördlich von Zehren entlang des Ketzerbaches ein neues Schutzgebiet festgesetzt. Es handelt sich um einen ganz neuen Typ eines Naturschutzgebietes, denn es ist kein einheitliches Gebilde, sondern besteht aus 15 Teilflächen.

\section{Naturschutzgebiet Täler südöstlich Lom- matzsch und gleichnamiges FFH-Gebiet}

\section{Größe: 140 Hektar}

Lage: 15 Teilflächen entlang des Ketzerbaches bis nördlich von Leuben und entlang des Käbschützbaches in den Gemeinden Diera-Zehren, Käbschütztal und der ehemaligen Gemeinde Leuben-Schleinitz sowie in Ortsteilen der Stadt Lommatzsch

Schutzzweck: Erhaltung, Wiederherstellung und Entwicklung von Lebensstätten, Biotopen und Lebensgemeinschaften wildlebender Tierund Pflanzenarten auf Trockenhängen mit überwiegend exothermen Lebensbedingungen an den Talflanken des Ketzerbaches zwischen Leuben und Schieritz und im unteren Käbschützbachtal mit seinem von Trockenhängen, Feuchtwiesen, Simsen- und Seggenrieden und dem naturnahen Käbschützbach kleinteilig geprägten Ensemble und funktionalen Komplex aus Biotopen und historischen Kulturlandschaftsbestandteilen innerhalb der ansonsten intensiv genutzten Agrarlandschaft des Mittelsächsischen Lößhügellandes.

„Der Käbschützgrund und das untere Ketzerbachtal gehören aufgrund ihrer bewegten Geomorphologie, ihrer großflächigen Xerothermstandorte und der noch in Resten erhaltenen Bestandteile der ansonsten weithin ausgeräumten Gefildelandschaft zu den landeskundlich, naturgeschichtlich und floristisch-faunistisch wertvollsten Gebieten des Elbhügellandes von besonderer Eigenart, regionaler Seltenheit und hervorragender Schönheit." So lautet der erste Satz der Würdigung für das Naturschutzgebiet. Die Auswahl der wertvollsten Flächen war nicht einfach, galt es doch, den Nutzungskonflikt mit den Flächennutzern zu minimieren. Der Weg zur Ausweisung des Schutzgebietes war lang und steinig. Bereits im 19. Jahrhundert erforschten Botaniker die bemerkenswerten Vorkommen wärmeliebender Pflanzen, und das Gebiet galt schon lange als das wertvollste und artenreichste Xerothermgebiet in Sachsen. 1958 wurden zwei Teilflächen als flächenhafte Naturdenkmale ausgewiesen.

Die Flora des Naturschutzgebiets zeigt sich mit mehr als 570 Gefäßpflanzenarten, von denen in Sachsen 23 Arten als vom Aussterben bedroht, 23 als stark gefährdet und 34 als gefährdet gel-

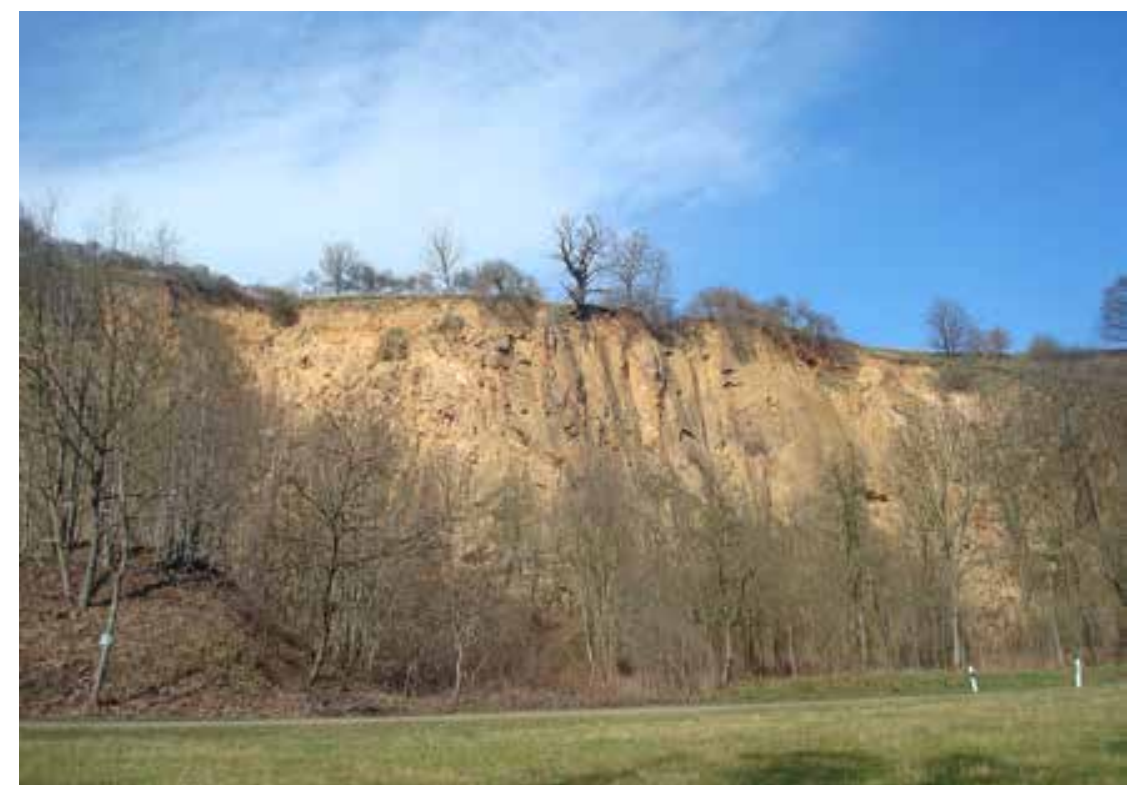

ten, als besonders artenreich. Hier können nicht alle aufgezeigt werden, denn die Liste ist sehr lang. Exemplarisch sollen hier nur wenige vom Aussterben bedrohte Arten genannt werden: Wiesen-Kuhschelle, Essigrose, Violette Königskerze, Bologneser Glockenblume und Acker-Schwarzkümmel. Die Tabelle der kartierten Insektenarten ist endlos. In der Würdigung des Naturschutzgebiets werden die Flächen als für Insekten bedeutsames Gebiet in Sachsen eingeschätzt. Bei den Wirbeltieren sei auf das Vorkommen der seltenen Glattnatter sowie vereinzelt der Ringelnatter verwiesen. Die Vögel sind mit 59 Brutvogelarten sehr zahlreich vertreten. Für das gesamte Naturschutzgebiet wertbestimmend ist das Brutvorkommen der als stark gefährdet geltenden Art Wendehals sowie der gefährdeten Arten Wespenbussard und Sperbergrasmücke. In den Eichen-Hainbuchenwald-Bereichen brütet der Rotmilan. Neun Fledermausarten nutzen das Schutzgebiet als Jagdhabitat, darunter Mopsfledermaus und Großes Mausohr. In den Bachläufen ist der seltene Fischotter heimisch und man kann hier die Spuren des Elbebibers finden.

Für den Naturfreund ist das Schutzgebiet wegen der zersplitterten Lage ohne Schutzgebietskarte schwer zu erkunden, auch weil außerhalb der Wege ein Betretungsverbot für Flächen besteht. Eine Wanderung von Zehren durch das Ketzerbachtal und durch den Käbschützgrund bis Görna ist äußerst empfehlenswert, denn die Natur kann man auch von den Wegen aus betrachten und erkunden. Außerdem gibt es noch vieles Reizvolles in den Dörfern zu entdecken.

Unweit von Mertitz liegt eine Fläche, die nicht zu einem Schutzgebiet gehört, aber ökologisch äußerst wertvoll ist. Die Fläche wurde schon
Ketzerbachtal bei Mertitz Foto: Frank Ende 


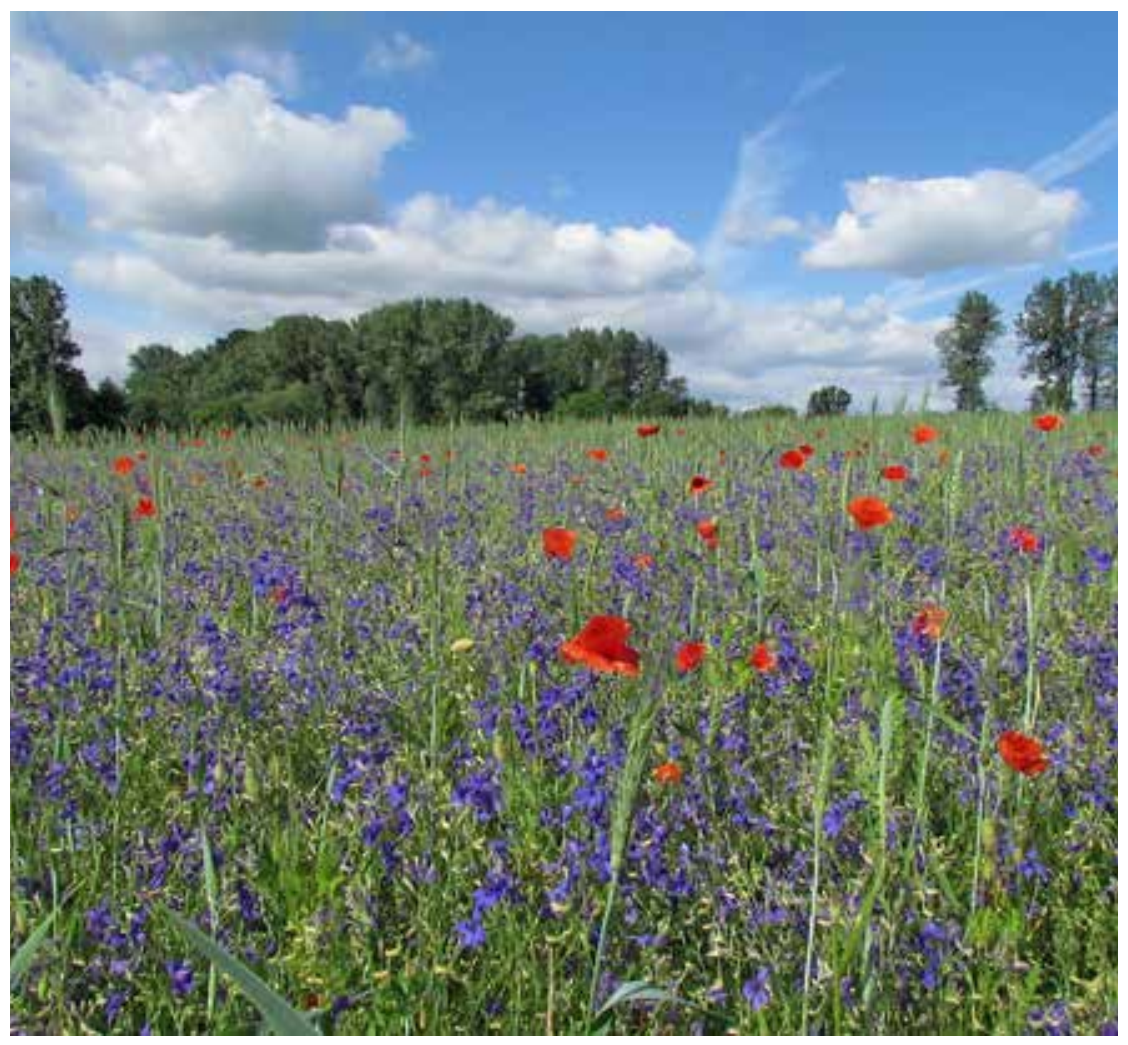

Schutzacker in Mertitz mit Klatschmohn und AckerRittersporn Foto: Frank Ende

Luchs im Garten in Nössige Foto: Frank Ende

Autor

Frank Ende Käbschütztal vor 1990 als Schutzacker für Ackerwildkräuter bewirtschaftet. Seit 2011 gehört sie dem Landesverein Sächsischer Heimatschutz. Sehenswert ist der Acker besonders im Mai und Juni. Er ist von Mertitz oder Schwochau über einen Feldweg zu Fuß zu erwandern. Der Acker-Rittersporn mit seiner blauen Farbe, der rote Klatschmohn und die weiße Kamille färben in dieser Zeit das Getreidefeld bunt. Daneben sind noch viele weitere seltene Ackerwildkräuter zu entdecken.

Auf meinem Weg durch die Naturschutzgebiete der Lommatzscher Pflege möchte ich noch auf einiges Sehenswerte verweisen, das mir - außerhalb der der großen Schutzgebiete - an meinen Wohnort Nössige begegnet ist. Fledermäuse kann man leicht in den frühen Sommerabendstunden beobachten, wenn sie ihre Schlafplätze in alten Gebäuden, zugänglichen Dächern oder höhlenreichen Bäumen verlassen und auf Nahrungssuche gehen. Bei mir in Nössige sind jedes Jahr zwischen zwei bis fünf Exemplare auch verschiedener Arten zu sehen. Die konkrete Artbestimmung ist den Fachleuten vorbehalten, aber mit Detektoren kann man auch als Laie die Art abschätzen.

Eine Naturbeobachtung der besonderen Art konnten wir 2012 machen: Meine Frau sah früh beim Füttern einen sichtlich hungrigen Luchs, der sich am Kaninchenstall zu schaffen machte. Dem „vor Angst schlotternden Fotografen“ gelangen jedoch einige leicht verwa- ckelte Beweisfotos. Wir hatten in der Nacht davor geglaubt, schon Vogelstimmen gehört zu haben, die sich am Tag darauf nach einer Internetrecherche als Rufe des Luchses herausstellten.

Im Jahr danach verirrte sich eine Schleiereule in ein Zimmer unseres Hauses. Erstaunlicherweise hatten wir noch nie Rufe dieser Eulenart gehört, sondern immer nur die der Waldkäuze, die in dem an unser Grundstück grenzenden Feldgehölz brüten. In den feuchten Mauerspalten von Gemäuern kann man den seltenen Tigerschnegel finden. Es ist eine sehr große Nacktschnecke mit einer markanten Streifenzeichnung. Diese sollte man keinesfalls zertreten.

Das Erscheinen des Frühlings macht sich bei uns mit morgendlichem schwätzendem Gesang vom Nachbargebäude bemerkbar. Ein Blick aus dem Schlafzimmerfenster: die Stare sind wieder da. Wenn dann alle bis zu 13 Brutpaare versammelt sind, werden wir jeden morgen nach Sonnenaufgang mit einem ohrenbetäubenden Gesang geweckt. Die Vorfahren auf diesem Hof hatten in der Giebelwand des Seitengebäudes kleine Hohlräume im Gemäuer geschaffen und davor einen kleinen Metallsteg als Sitzstange angebracht. Diese Höhlen nutzen die Stare für ihr Brutgeschäft von ein bis zwei Bruten jährlich. Im August sammeln sich die Jungstare zu gewaltigen Schwärmen, die man vielfach in den Dörfern beobachten kann.

Der Autor wollte mit dieser Beschreibung einiger weniger Sehenswürdigkeiten der Natur der Lommatzscher Pflege die Anregung zum Selbsterkunden der Natur in der Landschaft, aber auch in den Dörfern geben.

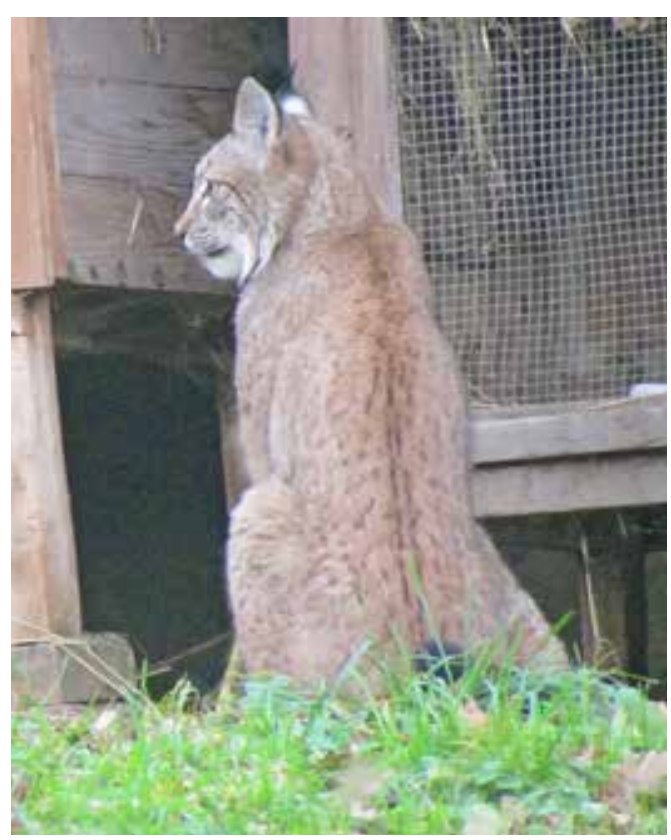

